\title{
Photonic integration in k-space: Enhancing the performance of photonic crystal dye lasers
}

Christiansen, Mads Brøkner; Kristensen, Anders; Xiao, Sanshui; Mortensen, Asger

Published in:

Applied Physics Letters

Link to article, DOI:

10.1063/1.3040686

Publication date:

2008

Document Version

Publisher's PDF, also known as Version of record

Link back to DTU Orbit

Citation (APA):

Christiansen, M. B., Kristensen, A., Xiao, S., \& Mortensen, A. (2008). Photonic integration in k-space: Enhancing the performance of photonic crystal dye lasers. Applied Physics Letters, 93(23), 231101.

https://doi.org/10.1063/1.3040686

\section{General rights}

Copyright and moral rights for the publications made accessible in the public portal are retained by the authors and/or other copyright owners and it is a condition of accessing publications that users recognise and abide by the legal requirements associated with these rights.

- Users may download and print one copy of any publication from the public portal for the purpose of private study or research.

- You may not further distribute the material or use it for any profit-making activity or commercial gain

- You may freely distribute the URL identifying the publication in the public portal 


\title{
Photonic integration in $k$-space: Enhancing the performance of photonic crystal dye lasers
}

\author{
Mads Brøkner Christiansen, ${ }^{1}$ Anders Kristensen, ${ }^{1, a)}$ Sanshui Xiao, ${ }^{2}$ and \\ Niels Asger Mortensen ${ }^{2}$ \\ ${ }^{1}$ Department of Micro and Nanotechnology, DTU Nanotech, Technical University of Denmark, \\ Building 345 East, DK-2800 Kongens Lyngby, Denmark \\ ${ }^{2}$ Department of Photonics Engineering, DTU Fotonik, Technical University of Denmark, \\ Building 345 West, DK-2800 Kongens Lyngby, Denmark
}

(Received 16 July 2008; accepted 12 October 2008; published online 8 December 2008)

\begin{abstract}
We demonstrate how two optical functionalities can be implemented in a single photonic crystal structure by carefully engineering dispersion in several different bands at several different wavelengths. We use the concept for optically pumped dye doped hybrid polymer band edge lasers and show how a rectangular photonic crystal lattice imprinted into the surface can provide both feedback for in-plane band edge lasing and couple pump light into the device plane, thus increasing the emitted intensity and lowering the lasing threshold by more than an order of magnitude. (C) 2008 American Institute of Physics. [DOI: 10.1063/1.3040686]
\end{abstract}

Photonic crystals ${ }^{1}$ (PhCs) provide new functionality to the dielectric materials they are made from. PhCs can exclude light from propagating, thus effectively functioning as a mirror, or the group velocity be reduced, thus increasing the effective interaction length between light and matter. ${ }^{2,3}$ In this letter we address multiband dispersion engineering as a means to integrate several different desired $\mathrm{PhC}$ functionalities in the same structure. By carefully structuring a dielectric material, several functionalities can be obtained, opposed to addressing each functionality, and then miniaturize and integrate individual components, as commonly done in engineering. As an example we demonstrate how a rectangular lattice can be designed to couple pump light more effectively into organic dye doped hybrid polymer band edge lasers from above, while the frequency of a group velocity slowdown, giving rise to band edge lasing, ${ }^{4}$ can be tuned independently.

A schematical drawing of a $\mathrm{PhC}$ band edge laser, with an inset atomic force microscope (AFM) image showing the surface structure, is seen in Fig. 1. It is a thin slab made of Ormocore, ${ }^{5}$ a type of ORMOCER hybrid polymer, ${ }^{6}$ doped with the organic laser dye Pyrromethene $597,{ }^{7}$ at a concentration of $5 \mu \mathrm{mol} / \mathrm{g}$ Ormocore. A rectangular lattice $\mathrm{PhC}$ is imprinted into the surface.

Laser light is emitted in the chip plane (polar angle $\theta$ $=90^{\circ}$ ) when the dye is optically pumped with a $Q$-switched $532 \mathrm{~nm}$ laser $(\approx 3 \mathrm{~mm}$ beam diameter). The pump light is incident at a polar angle close to $0^{\circ}$ and with an azimuthal angle $\phi=0^{\circ}$, corresponding to the $\Gamma X$ direction of the crystal lattice. The emitted light was collected with a multimode optical fiber [visible in Fig. 3(e)] and analyzed with an Ocean Optics HR4000 fixed grating spectrometer with a resolution of $\approx 0.035 \mathrm{~nm}$.

The lasers are fabricated in parallel by combined nanoimprint and photolithography ${ }^{8,9}$ on a $10 \mathrm{~cm}$ glass substrate, using an EVG 520HE nanoimprinter $(10 \mathrm{kN}, 5 \mathrm{~min}$, room temperature). After imprinting the stamp/substrate

\footnotetext{
${ }^{\text {a) }}$ Author to whom correspondence should be addressed. Electronic mail: anders.kristensen@nanotech.dtu.dk. URL: www.nanotech.dtu.dk/ak.
}

stack is UV flood exposed for $60 \mathrm{~s}$ at $9 \mathrm{~mW} / \mathrm{cm}^{2}$ in an UV aligner and postexposure baked at a $90{ }^{\circ} \mathrm{C}$ hotplate for 2 min, prior to stamp separation. Finally, unexposed Ormocore is dissolved in Ormodev $^{5}$ (180 s), followed by cleaning in isopropanol (30 s) and drying.

A typical TE band diagram for a rectangular lattice two dimensional (2D) $\mathrm{PhC}$ is shown in Fig. 2, which also shows the real-space lattice and the irreducible Brillouin zone. In a conventional (unshifted) distributed feedback laser, which is, in fact, a one dimensional $\mathrm{PhC}$ band edge laser, the surface grating is used only to lower the group velocity in a guided mode at the desired lasing frequency $\left(\omega_{l}\right)$. This functionality is provided by making the first and second bands $(i=1$ and $i=2$ ) flatten at the desired laser wavelength at the $P$ point in the Brillouin zone. The requirement can be stated as

$$
\omega_{i}(P)=\omega_{l},\left.\quad \frac{\partial \omega}{\partial k}\right|_{P}=0, \quad i=1 \text { or } 2 .
$$

Modeling was done by finding fully vectorial eigenmodes of Maxwell's equations with periodic boundary conditions by preconditioned conjugate-gradient minimization of the block Rayleigh quotient in a planewave basis, using a freely available software package. ${ }^{10}$ Modeling was done in 2D, using effective refractive indices of infinitely wide slab waveguides with the two heights of the laser $\mathrm{PhC}$ structure. The exact height of the device was calculated

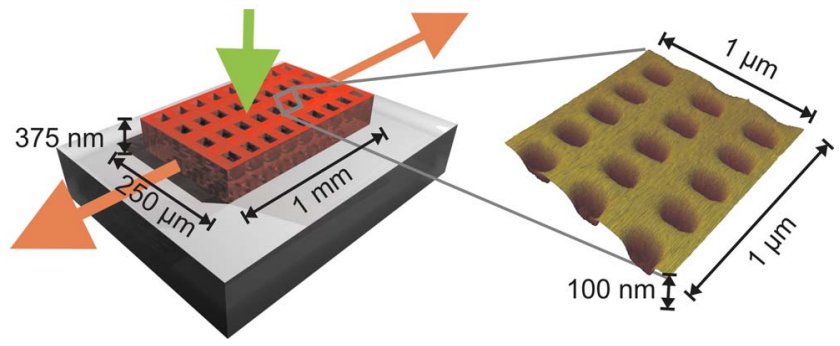

FIG. 1. (Color online) Illustration of the laser design with inset AFM image of a surface section. The lasers are made of Pyrromethene 597 dye doped Ormocore on a glass substrate. The green arrow indicates pump light, and the orange arrows indicate laser emission in the chip plane. 
(a)

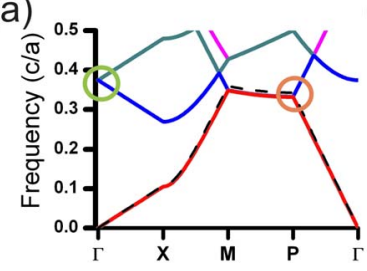

(b)

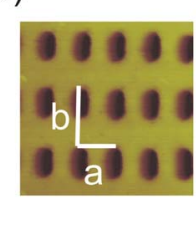

(c)

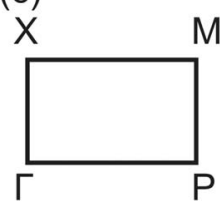

FIG. 2. (Color online) (a) Typical band diagram for the rectangular lattice lasers. The point giving rise to laser oscillations is circled in orange and the area used for pumping is circled in green. Above the dashed black curve the optical modes are leaky. (b) AFM image of the real-space structure with the $a$ and $b$ grating constants indicated. All devices were fabricated with $a$ $=200 \mathrm{~nm}$, while $b$ values ranged from 351 to $358 \mathrm{~nm}$. (c) The irreducible Brillouin zone.

from the laser emission characteristics and bulk index, calculated from Cauchy coefficients provided by micro resist technology $\mathrm{GmbH}$. The calculated height fitted well with stylus profiler measurements.

In a single pass pump scheme, homogeneous pumping requires the absorption length of the pump light to be substantially longer than the interaction length with the active material, thus implying a low external quantum efficiency. This issue can be addressed by designing the $\mathrm{PhC}$ in a way that the second $(i=2)$ or third $(i=3)$ band intersects with the pump light frequency $\left(\omega_{p}\right)$ very close to the $\Gamma$ point in the band structure, enabling light to be coupled effectively into the chip plane in a delocalized Bloch state. This requirement can be stated as

$$
\omega_{i}(\Gamma) \approx \omega_{p}, \quad i=2 \text { or } 3 .
$$

By tuning the $a$ and $b$ lattice constants in the rectangular lattice, the dispersion at the $P$ and $\Gamma$ points used can be tuned independently. To illustrate how the pump properties change with the long lattice constant, we have fabricated devices with different $b$ lattice constants, ranging from 351 to $358 \mathrm{~nm}$, while in the other direction, the lattice constant is fixed to $a=200 \mathrm{~nm}$. We can now look at the calculated band structure for each device near the $\Gamma$ point and determine the in-plane wavenumber $k_{\|}$of the appropriate band where it intersects with the frequency of the pump laser. The optimum pump angle $(\theta)$ can thus, from a simple geometrical consideration, be calculated as

$$
\theta=\arcsin \left(\frac{k_{\|}}{k_{p}}\right),
$$

where $k_{p}=\omega_{p} / c$ is the pump wavenumber. In Fig. 3 panel (a) the laser output signal as a function of pump angle is shown for three different lasers with different $b$ grating constants. Since the devices have inversion symmetry with respect to the lattice vectors, the resonance condition is fulfilled at both positive and negative angles. In panel (b) of Fig. 3 the measured resonance angle is plotted as a function of $b$ lattice period, along with theoretical calculations. It is evident how the resonance angle goes to $0^{\circ}$ and back because the $\Gamma$ point in the band structure is scanned across the pump frequency. We attribute the deviations at larger angles to our simplified description of the $\mathrm{PhC}$ as an infinitely high 2D system rather than a structured thin film. Figure 3 panel (c) shows threshold curves for the same laser $(b=355 \mathrm{~nm})$ pumped at two different angles. The threshold (not emphasized in the figure) drops an order of magnitude from (a)

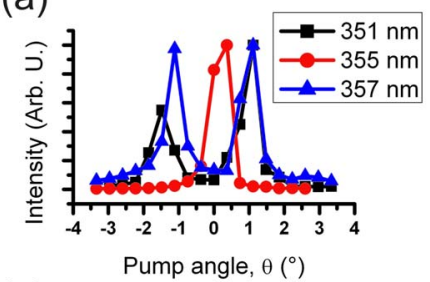

(c)

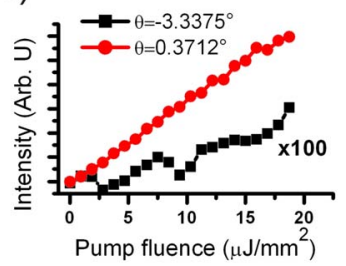

(b)

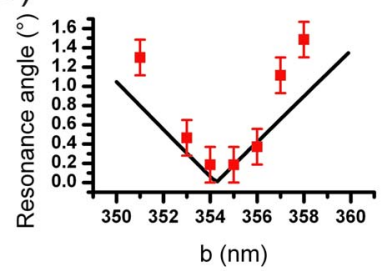

(d)

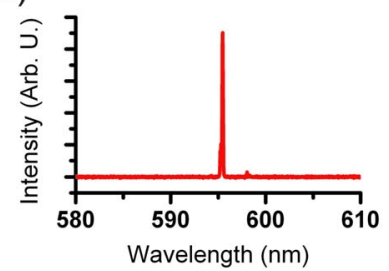

(e)

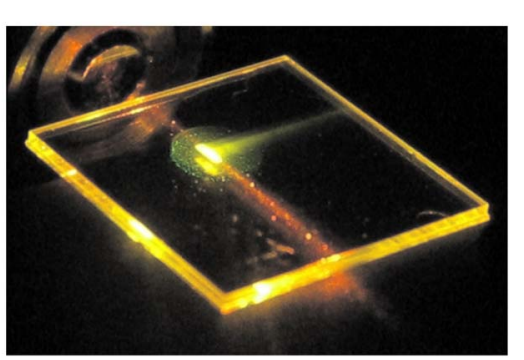

FIG. 3. (Color online) (a) Output intensity as a function of pump angle $(\theta)$ for three devices with different $b$ grating constants. (b) Calculated (black curve) and measured (red squares) resonance pump angles as a function of $b$ grating constant. (c) Curves showing output as a function of pump intensity for the same $b=355 \mathrm{~nm}$ device pumped at two different angles. The lasing threshold when measuring at resonance is $470 \mathrm{~nJ} / \mathrm{mm}^{2}, 16$ times lower than the off-resonance threshold, and the difference in maximum measured output signal is almost a factor of 200 . Note that the lower trace has been magnified by a factor of 100. (d) The narrow laser spectrum from a $b$ $=353 \mathrm{~nm}$ device pumped at $4 \mu \mathrm{J} / \mathrm{mm}^{2}$. (e) A photo of a chip with an operating laser. From the ends of the laser, orange light is emitted, and perpendicular to this, a green pump laser beam is seen, which is scattered into the chip plane by the $\mathrm{PhC}$. The measurement fiber is also seen in the top left corner.

$7.9 \mu \mathrm{J} / \mathrm{mm}^{2}$ to $470 \mathrm{~nJ} / \mathrm{mm}^{2}$ as the resonance angle is hit, but clearer from the figure is that the maximum output signal is also increased by almost a factor of 200. A typical laser spectrum for a device pumped at $4 \mu \mathrm{J} / \mathrm{mm}^{2}$ is seen in Fig. 3 panel (d). When the laser is pumped at approximately eight times the threshold, as in this case, the output is spectrally narrow. The laser oscillations primarily take place at the band edge of the second band $i=2$, since the dye provides larger gain at this wavelength, but another very small peak, which is attributed to competing lasing in the first band $(i=1)$, can barely be seen in the spectrum, a few nanometers redshifted, compared to the main peak. When the lasers are pumped far above threshold, this signal also grows substantially due to spatial hole burning. The two effects of the PhC are clearly visible when the lasers are operated. Figure 3 panel (e) is a photo, taken through an optical filter, of a laser operating under resonance pump conditions. From the ends orange $(\approx 595 \mathrm{~nm})$ laser light is emitted, but perpendicular to this, a green light beam is also seen. This is unabsorbed pump laser light, which is scattered almost $90^{\circ}$ into the chip plane by the $\mathrm{PhC}$.

The functionalities of the lasers described above could also have been achieved with two separate narrow band Fabry-Pérot cavities; one for the lasing oscillations and one 
for the efficient pumping. However, by designing the functionality in reciprocal space rather than real space, it is only necessary to use one low index material, in this case Pyrromethene 597 doped Ormocore, and the fabrication is still a low cost single step parallel process, irrespective of the number of functionalities achieved through the $\mathrm{PhC}$ structure. One way of simplifying laser operation is to make a flat band at the pump frequency at the $\Gamma$ point, i.e., lower $\partial \omega /\left.\partial k\right|_{\Gamma}$. This would broaden the acceptance angle for achieving resonance pumping, but lower the fabrication tolerance, since the band edge had to be aligned completely with the pump frequency.

Optical integration in $k$-space, as demonstrated here, is not only applicable to low cost low index nanostructured lasers, ${ }^{11-13}$ but could also impact photonic integration in general. It could, e.g., be applied to low and high index PhCs for sensing and optofluidics ${ }^{14-17}$ as well as other types of adaptive optics, ${ }^{18}$ for slow light engineering, ${ }^{19}$ collimation, and hyperlensing $^{20-23}$ or for highly confined optical cavities. $^{24}$ The method could be extended to provide entirely new multifunctional materials, as metamaterials hold promise for. ${ }^{25}$ Here, we have used an elementary PhC to illustrate the concept of $k$-space integration, but through careful design of $\mathrm{PhC}$ structure, we believe that material properties can be designed to fit many different applications, where light is handled in different propagation directions, different frequencies, and/or at different group velocities.

The support of the Toubro Foundation and the ECfunded projects NaPa (Contract No. NMP4-CT-2003500120) and NaPANIL (Contract No. 214249) is gratefully acknowledged. Sanshui Xiao is financially supported by the Danish Research Council for Technology and Production Sciences (Grant No. 274-07-0379).

${ }^{1}$ J. D. Joannopoulos, R. D. Meade, and J. N. Winn, Photonic Crystals: Molding the Flow of Light (Princeton University Press, Princeton, NJ, 1995).
${ }^{2}$ M. Soljacic, S. G. Johnson, S. H. Fan, M. Ibanescu, E. Ippen, and J. D. Joannopoulos, J. Opt. Soc. Am. B 19, 2052 (2002).

${ }^{3}$ N. A. Mortensen and S. Xiao, Appl. Phys. Lett. 90, 141108 (2007).

${ }^{4}$ J. P. Dowling, M. Scalora, M. J. Bloemer, and C. M. Bowden, J. Appl. Phys. 75, 1896 (1994)

${ }^{5}$ Commercially available from micro resist technology $\mathrm{GmbH}$, Berlin, Germany, www.microresist.de.

${ }^{6}$ ORMOCER is a registered trademark of Fraunhofer-Gesellschaft zur Förderung der Angewandten Forschung e.V., Germany.

${ }^{7}$ Pyrromethene 597, CAS No. 137829-79-9, acquired from Exciton Inc., www.exciton.com.

${ }^{8}$ X. Cheng and L. J. Guo, Microelectron. Eng. 71, 288 (2004).

${ }^{9}$ M. B. Christiansen, M. Schøler, and A. Kristensen, Opt. Express 15, 3931 (2007)

${ }^{10}$ S. G. Johnson and J. D. Joannopoulos, Opt. Express 8, 173 (2001).

${ }^{11}$ D. Pisignano, L. Persano, G. Gigli, P. Visconti, T. Stomeo, M. De Vittorio, G. Barbarella, L. Favaretto, and R. Cingolani, Nanotechnology 15, 766 (2004).

${ }^{12}$ A. Vasdekis, G. Turnbull, I. Samuel, P. Andrew, and W. Barnes, Appl. Phys. Lett. 86, 161102 (2005).

${ }^{13}$ V. Reboud, P. Lovera, N. Kehagias, M. Zelsmann, C. Schuster, F. Reuther, G. Gruetzner, G. Redmond, and C. Torres, Appl. Phys. Lett. 91, 151101 (2007)

${ }^{14}$ M. Lončar, A. Scherer, and Y. Qiu, Appl. Phys. Lett. 82, 4648 (2003).

${ }^{15}$ M. Adams, M. Lončar, A. Scherer, and Y. Qiu, IEEE J. Sel. Areas Commun. 23, 1348 (2005).

${ }^{16}$ C. L. C. Smith, D. K. C. Wu, M. W. Lee, C. Monat, S. Tomljenovic-Hanic, C. Grillet, B. J. Eggleton, D. Freeman, Y. Ruan, S. Madden, and B. Luther-Davies, Appl. Phys. Lett. 91, 121103 (2007).

${ }^{17}$ F. Arango, M. Christiansen, M. Gersborg-Hansen, and A. Kristensen, Appl. Phys. Lett. 91, 223503 (2007).

${ }^{18}$ Y. A. Vlasov, M. O'Boyle, H. F. Hamann, and S. J. McNab, Nature (London) 438, 65 (2005).

${ }^{19}$ T. Baba and D. Mori, J. Phys. D: Appl. Phys. 40, 2659 (2007).

${ }^{20}$ H. Kosaka, T. Kawashima, A. Tomita, M. Notomi, T. Tamamura, T. Sato, and S. Kawakami, Appl. Phys. Lett. 74, 1212 (1999).

${ }^{21}$ J. Witzens, M. Lončar, and A. Scherer, IEEE J. Sel. Top. Quantum Electron. 8, 1246 (2002)

${ }^{22}$ L. J. Wu, M. Mazilu, and T. F. Krauss, J. Lightwave Technol. 21, 561 (2003).

${ }^{23}$ D. W. Prather, S. Y. Shi, D. M. Pustai, C. H. Chen, S. Venkataraman, A. Sharkawy, G. J. Schneider, and J. Murakowski, Opt. Lett. 29, 50 (2004).

${ }^{24}$ S. Noda, A. Chutinan, and M. Imada, Nature (London) 407, 608 (2000).

${ }^{25}$ A. Lakhtakia and T. G. Mackay, Opt. Photonics News 18, 32 (2007). 